VoL. 53 (1996) [33-37]

\title{
ON THE CONVEXITY OF STAMPFLI'S NUMERICAL RANGE
}

\author{
J.O. Agure
}

This paper investigates a certain type of numerical range introduced by Stampfli. In particular, we investigate the convexity of this set of elements of operators on Hilbert spaces and its relationship to the algebra numerical range implemented by elements of a $W^{*}$-algebra.

\section{INTRODUCTION}

Let $\mathfrak{S}$ be a complex Hilbert space, $\mathfrak{L}(\mathfrak{S})$ the set of bounded linear operators on H. Stampfli [4] defined the $\delta$-numerical range, $W_{\delta}(T)$, as the set

$$
W_{6}(T)=\text { closure }\{\langle T x, x\rangle:\|x\|=1 \text { and }\|T x\| \geqslant \delta\} .
$$

Having introduced this set, he wondered whether for any $T \in \mathfrak{B}(\mathfrak{X})$, the set $W_{\delta}(T)$ is convex. (Here $\mathfrak{B}(\mathfrak{X})$ is the set of bounded linear operators on a Banach space $\mathfrak{X}$.) We shall solve this problem for $\mathfrak{L}(\mathfrak{S})$ using an idea of Dekker, Bonsall and Duncan, [2]. We then proceed to introduce the set $V_{\delta}(T)$, where

$$
V_{\delta}(T)=\operatorname{closure}\left\{f(T): f(I)=\|f\|=1 \text { and } f\left(T^{*} T\right) \geqslant \delta^{2}\right\}
$$

is the algebra $\delta$-numerical range for an element $T$ of a unital $W^{*}$-algebra $\mathfrak{U}$. We conclude by proving that the sets $W_{\delta}(T)$ and $V_{\delta}(T)$ are actually equal.

We remind the reader that a subset $G$ of $\mathbb{C}$ is said to be connected if it cannot be split into two nonempty open sets. The following lemma shows when the set is connected.

LEMma 1.1. Let $T$ be a self adjoint element of $\mathfrak{L}(\mathfrak{S})$ and let

$$
E=\{x \in \mathfrak{S}:\|x\| \text { and }\langle T x, x\rangle=0\}
$$

Then $E$ is arcwise connected.

The proof of this lemma can be found in Bonsall and Duncan, [2]. We shall now prove the convexity of the set $W_{\delta}(T)$ for any $T \in \mathfrak{L}(\mathfrak{S})$. We would like to point out that the proof of this theorem also appears in the author's Ph.D. thesis, Agure [1].

Received 14 March 1995

Copyright Clearance Centre, Inc. Serial-fee code: 0004-9729/96 \$A2.00+0.00. 
THEOREM 1.2. $W_{\delta}(T)$ is a convex set.

Proof: Let $l$ be a straight line in $\mathbb{C}$. To prove that the set $W_{\delta}(T)$ is convex, it is enough to show that the intersection $W_{\delta}(T) \cap l$ is a connected set. If $T=T_{1}+i T_{2}$, $T_{i}=T_{i}^{*}, i=1,2$ and $L=a T_{1}+b T_{2}+c I$, (where $I$ is the identity operator) and $a$, $b, c$ are constants, then it is clear that $L$ is a self adjoint operator. Consider the set $E$ given by

$$
E=\left\{x \in \mathfrak{S}:\|x\|=1,\left\|\left(T_{1}+i T_{2}\right) x\right\| \geqslant \delta \text { and }\langle L x, x\rangle=0\right\} .
$$

Assume that $E$ is nonempty. We shall show that $E$ is arcwise connected. To do that choose any two vectors $y_{1}, y_{2}$ in $E$. Note that there is no loss of generality in assuming that the vectors $y_{1}$ and $y_{2}$ are linearly independent elements of $E$. Clearly if $y \in E$, then for any $\alpha \in \mathbb{R}, y e^{i \alpha} \in E$ and $y$ is joined to $y e^{i \alpha}$ by an $\operatorname{arc} t$ that maps $[0, \alpha]$ to $E, t \rightarrow e^{i \alpha t} y$. Next choose $\alpha \in \mathbb{R}$ such that $e^{i \alpha}\left\langle L y_{1}, y_{2}\right\rangle$ is purely imaginary and take $\widehat{y}_{1}=y_{1} e^{i \alpha}, \alpha \in \mathbb{R}$. Secondly take $\beta$ so that

$$
\Re \mathrm{e}\left\langle\left(T^{*} T-\delta^{2}\right) \widehat{y}_{1}, e^{i \beta} y_{2}\right\rangle \geqslant 0
$$

and set $\widehat{y}_{2}=e^{i \beta} y_{2}$. We are left with the task of showing that $\widehat{y}_{1}$ can be joined to $\widehat{y}_{2}$ by an arc in $E$. It is clear that $\left\langle L \widehat{y}_{1}, \widehat{y}_{2}\right\rangle$ will be purely imaginary. Now with $0 \leqslant s \leqslant 1$, let $x(s)$ and $p(s)$ be given by

$$
x(s)=(1-s) \widehat{y}_{1}+s \widehat{y}_{2}, \quad p(s)=\frac{1}{\|x(s)\|} x(s) .
$$

We shall show that the arc $p(s)$ is in the set $E$ by first proving that $\langle L p(s), p(s)\rangle=$ 0 , and then $\|T p(s)\| \geqslant \delta$. Now

$$
\begin{aligned}
\langle L x(s), x(s)\rangle & =\left\langle L(1-s) \widehat{y}_{1}+s \widehat{y}_{2},(1-s) \widehat{y}_{1}+s \widehat{y}_{2}\right\rangle \\
& =(1-s)^{2}\left\langle L \widehat{y}_{1}, \widehat{y}_{1}\right\rangle+(1-s) s\left(\left\langle L \widehat{y}_{1}, \widehat{y}_{2}\right\rangle+\left\langle L \widehat{y}_{2}, \widehat{y}_{1}\right\rangle\right)+s^{2}\left\langle L \widehat{y}_{2}, \widehat{y}_{2}\right\rangle \\
& =(1-s) s\left\{\left\langle L \widehat{y}_{1}, \widehat{y}_{2}\right\rangle+\left\langle L \widehat{y}_{2}, \widehat{y}_{1}\right\rangle\right\}=2(1-s) s \Re \mathrm{e}\left\langle L \widehat{y}_{1}, \widehat{y}_{2}\right\rangle=0
\end{aligned}
$$

as $\left\langle L \widehat{y}_{1}, \widehat{y}_{2}\right\rangle$ is purely imaginary. Therefore it is clear that $\langle L p(s), p(s)\rangle=0$. Also

$$
\begin{aligned}
\|T p(s)\|^{2}= & \frac{1}{\|x(s)\|^{2}}\|T x(s)\|^{2}=\frac{1}{\|x(s)\|^{2}}\langle T x(s), T x(s)\rangle \\
= & \frac{1}{\|x(s)\|^{2}}\left\{(1-s)^{2}\left\|T \widehat{y}_{1}\right\|^{2}+s^{2}\left\|T \widehat{y}_{2}\right\|^{2}+2(1-s) s \delta^{2} \Re \mathrm{e}\left\langle\widehat{y}_{1}, \widehat{y}_{2}\right\rangle\right. \\
& \left.+2(1-s) s \Re \mathrm{e}\left\langle T \widehat{y}_{1}, T \widehat{y}_{2}\right\rangle-2 \delta^{2}(1-s) s \Re \mathrm{e}\left\langle\widehat{y}_{1}, \widehat{y}_{2}\right\rangle\right\} \\
\geqslant & \frac{1}{\|x(s)\|^{2}}\left\{\|x(s)\|^{2} \delta^{2}+2(1-s) s \delta^{2} \Re \mathrm{e}\left\langle\widehat{y}_{1}, \widehat{y}_{2}\right\rangle+\right. \\
& \left.2(1-s) s \Re \mathrm{e}\left\langle T \widehat{y}_{1}, T \widehat{y}_{2}\right\rangle-2 \delta^{2}(1-s) s \Re \mathrm{e}\left\langle\widehat{y}_{1}, \widehat{y}_{2}\right\rangle\right\} \\
= & \delta^{2}+\frac{1}{\|x(s)\|^{2}}\left\{2(1-s) s \Re \mathrm{e}\left\langle\left(T^{*} T-\delta^{2}\right) \widehat{y}_{1}, \widehat{y}_{2}\right\rangle\right\} .
\end{aligned}
$$


And we see that $\|T p(s)\| \geqslant \delta$ by virtue of $\left(^{*}\right)$. We therefore conclude that the set $E$ is connected and the required arc is $p(s)$. Now define the mapping $\pi$ on $E$ by

$$
\pi(x)=\left\langle T_{1} x, x\right\rangle+i\left\langle T_{2} x, x\right\rangle, \forall x \in E .
$$

Then it is clear that $\pi(x) \in l$ and $\pi(x) \in W_{\delta}(T)$. So $W_{\delta}(T) \cap l=\pi(x)$. This mapping is also continuous. Since $E$ is connected and the mapping $\pi$ is continuous we conclude that $\pi(E)$ is connected and hence $W_{\delta}(T)$ is convex.

We shall now introduce the algebra numerical range. Suppose $\mathfrak{U}$ is a unital $W^{*}$ algebra, $\mathfrak{U}^{*}$ its dual and $E(\mathfrak{U})$ the set of states on $\mathfrak{U}$, that is

$$
E(\mathfrak{U})=\left\{f \in \mathfrak{U}^{*}: f(I)=1=\|f\|\right\} .
$$

Then for any $T$ in $\mathfrak{U}$ we define $V_{6}(T)$ by

$$
V_{\delta}(Y)=\operatorname{closure}\left\{f(T): f \in E(\mathfrak{U}) \text { and } f\left(T^{*} T\right) \geqslant \delta^{2}\right\} .
$$

THEOREM 1.3. $V_{\delta}(T)$ is a convex set.

Proof: Let $\lambda_{1}$ and $\lambda_{2} \in V_{\delta}(T)$. Then there exists states $g_{1}$ and $g_{2}$ on $\mathfrak{U}$ such that $g_{1}(T)=\lambda_{1}, g_{1}\left(T^{*} T\right) \geqslant \delta^{2}$ and $g_{2}(T)=\lambda_{2}, g_{2}\left(T^{*} T\right) \geqslant \delta^{2}$. Now for $0 \leqslant \alpha \leqslant 1$, let

$$
g(T)=\alpha g_{1}(T)+(1-\alpha) g_{2}(T) .
$$

The problem is to show that $g(T) \in V_{\delta}(T)$. It is clear that $g$ is linear and that

$$
\begin{aligned}
g\left(T^{*} T\right) & =\alpha g\left(T^{*} T\right)+(1-\alpha) g\left(T^{*} T\right) \\
& \geqslant \alpha \delta^{2}+(1-\alpha) \delta^{2}=\delta^{2}>0 .
\end{aligned}
$$

Therefore $g$ is a positive linear functional and since

$$
g(I)=\alpha g_{1}(I)+(1-\alpha) g_{2}(I)=1, \text { and }\|g\|=1,
$$

$g$ is in fact a state on $\mathfrak{U}$. Hence $g(T) \in V_{\delta}(T)$.

Having proved that both sets $W_{\delta}(T)$ and $V_{\delta}(T)$ are convex we shall now show that those two sets are actually equal.

THEOREM 1.4. For any $T \in \mathfrak{L}(\mathfrak{S}), W_{\delta}(T)=V_{\delta}(T)$.

Proof: It is easy to show that $W_{\delta}(T) \subseteq V_{\delta}(T)$. To prove the converse, we shall assume that $\lambda \in V_{\delta}(T)$ and $\lambda \notin W(T)$ and deduce a contradiction. Since $\lambda \in V_{\delta}(T)$, it follows that there exists a state $f$ in $\mathfrak{U}^{*}$ such that $f(T)=\lambda$ and $f\left(T^{*} T\right) \geqslant \delta^{2}$. $W_{\delta}(T)$ is convex. By rotating $T$, we may assume that

$$
\Re \text { e } W_{\delta}(T) \leqslant \Re e \lambda-\alpha, \quad \alpha>0 .
$$


Let

$$
G=\{x \in \mathcal{S},\|x\|=1 \text { and } \Re \mathrm{e}\langle T x, x\rangle \geqslant \Re \mathrm{e} \lambda-\alpha / 2, \alpha>0\}
$$

and

$$
\eta=\sup \{\|T x\|: x \in G\} .
$$

Then $\eta<\delta$. The set $G$ is nonempty because if it is not, then for all $x \in \mathfrak{S},\|x\|=1$, we shall have

$$
\Re \mathrm{e}\langle T \boldsymbol{x}, \boldsymbol{x}\rangle<\Re \mathrm{e} \lambda-\alpha / 2, \quad \alpha>0 .
$$

But since $f$ is a weak*-limit of convex combinations of vector states

$$
\forall \varepsilon>0 \exists N=N(\varepsilon): \forall n>N, \quad\left|f_{n}(T)-f(T)\right|<\varepsilon .
$$

Also we can find $M=M(\varepsilon)$ such that for all $n>M$ the following inequality will hold:

$$
\left|f_{n}\left(T^{*} T\right)-f\left(T^{*} T\right)\right|<\varepsilon .
$$

Take $\epsilon<\min \left\{\alpha / 2,\left(\delta^{2}-2 \delta \eta\right) / 2\right\}$ and $n>\max (N, M)$. Since

$$
f_{n}(T)=\sum_{i=1}^{n} \alpha_{i} \omega_{z_{i}}(T)=\sum_{i=1}^{n} \alpha_{i}\left\langle T x_{i}, x_{i}\right\rangle
$$

for $0 \leqslant \alpha_{i} \leqslant 1$, and $\sum_{i=1}^{n} \alpha_{i}=1$, we have

$$
\begin{aligned}
\Re \text { e } f_{n}(x) & =\Re e \sum_{i=1}^{n} \alpha_{i} \omega_{x_{i}}(T)=\Re e \sum_{i=1}^{n} \alpha_{i}\left\langle T x_{i}, x_{i}\right\rangle \\
& =\sum_{i=1}^{n} \alpha_{i} \Re e\left\langle T x_{i}, x_{i}\right\rangle<\Re e \lambda-\alpha / 2
\end{aligned}
$$

But $f_{n}(x)>f(T)-\varepsilon$ and therefore we see that $\Re$ e $f_{n}(x)>\Re e \lambda-\varepsilon$, implying that $\varepsilon>\alpha / 2$, which is a contradiction.

Now for all $x_{i} \in G$, we have $\left\|T x_{i}\right\| \leqslant \eta$. Since also $f\left(T^{*} T\right)<f_{n}\left(T^{*} T\right)+\varepsilon$ and $\delta^{2} \leqslant f\left(T^{*} T\right)$ we obtain

$$
\begin{aligned}
\delta^{2} \leqslant f\left(T^{*} T\right)<f_{n}\left(T^{*} T\right)+\varepsilon & =\sum_{i=1}^{n} \alpha_{i}\left\|T x_{i}\right\|^{2}+\varepsilon<\eta^{2}+\left(\delta^{2}-2 \delta \eta\right) / 2 \\
& =\eta^{2}+1 / 2(\delta-\eta)^{2}-\eta^{2} / 2 \\
& =\eta^{2} / 2+1 / 2(\delta-\eta)^{2}<\delta^{2}
\end{aligned}
$$

which is a contradiction. So we see that $\lambda \notin W_{\delta}(T)$ implying that $\lambda \notin V_{\delta}(T)$. Hence $\lambda \in V_{\delta}(T)$ implies that $\lambda \in W_{\delta}(T)$, and so $V_{\delta}(T) \subseteq W_{\delta}(T)$. 


\section{REFERENCES}

[1] J.O. Agure, 'On the numerical ranges and norms of derivations', Ph.D. Thesis (University of Birmingham, England, 1992).

[2] F.F. Bonsall and J. Duncan, Numerical Ranges II (Cambridge University Press, New York, 1973).

[3] J.G. Stampfli, 'Norms of derivations', Pacific Journal of Mathematics 33 (1970), 737-747.

Kenyatta University

Mathematics Department

PO Box 43844

Nairobi

Kenya 